Revista Ibero-Americana de Ciências Ambientais

Ibero-American Journal of Environmental Sciences

Edição Especial do Congresso de Gestão Ambiental do Baixo Amazonas

(CONGABA) Ago 2018 - v.9 - n.6

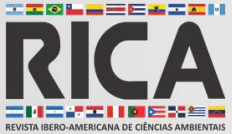

ISSN: 2179-6858

This article is also available online at: www.sustenere.co

\title{
Distribuição de larvas de Brycon amazonicus (PISCES: BRYCONIDAE) no entorno da Reserva de Desenvolvimento Sustentável Mamirauá: base ecológica para manejo
}

\begin{abstract}
A conservação dos recursos pesqueiros no médio Amazonas está relacionada ao conhecimento acerca dos seus estoques pesqueiros mais importantes, incluindo também o conhecimento sobre os padrões de recrutamento e desenvolvimento dos primeiros estágios de vida das espécies de maior importância econômica para a região. $O$ presente trabalho amplia o conhecimento ecológico sobre a distribuição das larvas de Brycon amazonicus (matrinxã) a fim de verificar a importância dos ambientes fluviais para o recrutamento biológico dessa espécie, auxiliando no manejo sustentável e na conservação desse recurso pesqueiro da Amazônia Central. Este trabalho foi realizado no entorno da Reserva de Desenvolvimento Sustentável do Mamirauá (RDSM) manejo sustentável e na conservação desse recurso pesqueiro da Amazônia Central. Este trabalho foi realizado no entorno da Reserva de Desenvolvimento Sustentável do Mamirauá (RDSM), arrastos horizontais na superfície e em profundidade $(8 \mathrm{~m})$, utilizando rede de plâncton $300 \mu \mathrm{m}$ em 16 estações de amostragem. Foram capturadas 310 larvas de matrinxã, sendo 148 indivíduos arrastos horizontais na superfície e em profundidade $(8 \mathrm{~m})$, utilizando rede de plâncton $300 \mu \mathrm{m}$ em 16 estações de amostragem. Foram capturadas 310 larvas de matrinxã, sendo 148 indivíduos coletados no Rio Solimões e 162 no Rio Japurá. Observou-se que não houve um padrão claro de distribuição entre os habitats amostrados; entretanto, foi constatado variação da abundância larval indicando distribuição espacial (estratos da coluna d'água) e temporal (ciclo diário), com as maiores abundâncias registradas na subsuperfície da água durante o período noturno. Larvas
registradas em estágios mais avançados de desenvolvimento refletem a importância da área de estudo para o recrutamento biológico, servindo como área de criação e alimentação para as larvas de matrinxã, sendo consideradas Habitats Essenciais para Peixes (EFHs) fundamentais para o manejo sustentável e conservação desse recurso pesqueiro da Amazônia Central.
\end{abstract}

Palavras-chave: Larvas de peixe; Matrinxã; Habitats essenciais; Manejo; Médio Solimões.

\section{Larval distribution of Brycon amazonicus (PISCES: BRYCONIDAE) around the Mamirauá Sustainable Development Reserve: an ecological basis for management}

\begin{abstract}
Conservation of fishery resources in the middle Amazon is related to knowledge of their most important fish stocks, including knowledge of recruitment and development patterns of early life of the most economically important species in the region. The present work expands the ecological knowledge about the distribution of Brycon amazonicus larvae (matrinxã) in order to verify the importance of fluvial environments for the biological recruitment of this species, helping in the sustainable management and conservation of this fishing resource of the Centra Amazon. This work was carried out around the Mamirauá Sustainable Development Reserve (RDSM) and the data obtained came from day and night collections carried out during the 2011 However, it was observed variation of larval abundance indicating spatial (water column strata) and temporal (daily cycle) distribution, with the highest abundances recorded in the water subsurface during the night period. Larvae recorded at more advanced stages of development reflect the importance of the study area for biological recruitment, serving as a breeding and feeding area for matrinxã larvae, and are considered essential fish habitat for sustainable management and conservation of this species fishing resource of the Central Amazon.
\end{abstract}

Keywords: Fish larvae; Matrinxã; Essential habitats; Management; Medium Solimões.

Ruineris Almada Cajado (iD)

Universidade Federal do Oeste Pará, Brasil http://lattes.cnpq.br/3589138303780787

http://orcid.org/0000-0002-7047-0302

ruineris.cajado@gmail.com

Lucas Silva de Oliveira (iD)

Universidade Federal do Oeste do Pará, Brasil http://lattes.cnpq.br/6700191077948533

http://orcid.org/0000-0002-1631-0498 lucasmdcpa@gmail.com

Cleidevania Cardoso de Oliveira (iD) Universidade Federal do Oeste do Pará, Brasil http://lattes.cnpq.br/4956595147053371 http://orcid.org/0000-0001-9677-3099 cleidevaniaoliver18@gmail.com
Silvana Cristina Silva da Ponte

Universidade Federal do Oeste do Pará, Brasi http://lattes.cnpq.br/1040146130702348 http://orcid.org/0000-0003-2818-166X silvanacristinasp@hotmail.com

Suzana Carla da Silva Bittencourt Universidade Federal do Pará, Brasi http://lattes.cnpq.br/2733502143762906 http://orcid.org/0000-0002-8162-1399 suzy_bitt@yahoo.com.br

Helder Lima de Queiroz (ic

Instituto de Desenvolvimento Sustentável Mamirauá, Brasil http://lattes.cnpq.br/3131281054700225

http://orcid.org/0000-0002-4425-3208 helder@mamiraua.org.br
Diego Maia Zacardi

Universidade Federal do Oeste do Pará http://lattes.cnpq.br/8348319991578546 http://orcid.org/0000-0002-2652-9477 dmzacardi@hotmail.com

DOI: 10.6008/CBPC2179-6858.2018.006.0010

Referencing this:

CAJADO, R. A.; OLIVEIRA, L. S.; OLIVEIRA, C. C.; PONTE, S. C. S.; BITTENCOURT, S. C. S.; QUEIROZ, H. L.; ZACARDI, D. M.. Distribuição de larvas de Brycon amazonicus (PISCES: BRYCONIDAE) no entorno da Reserva de Desenvolvimento Sustentável Mamirauá: base ecológica para manejo. Revista Ibero Americana de Ciências Ambientais, v.9, n.6, p.78-87, 2018. DOI: http://doi.org/10.6008/CBPC2179$\underline{6858.2018 .006 .0010}$ 
Distribuição de larvas de Brycon amazonicus (PISCES: BRYCONIDAE) no entorno da Reserva de Desenvolvimento Sustentável Mamirauá: base ecológica para manejo

CAJADO, R. A.; OLIVEIRA, L. S.; OLIVEIRA, C. C.; PONTE, S. C. S.; BITTENCOURT, S. C. S.; QUEIROZ, H. L.; ZACARDI, D. M.

\section{INTRODUÇÃO}

O peixe é um dos recursos de maior importância na Amazônia Central. Sua comercialização gera expressivas contribuições para a economia local, além de ser a principal fonte de proteína animal para os ribeirinhos (SANTOS et al., 2005). Dessa forma, a conservação destes recursos é fundamental para garantir a segurança econômica e alimentar da região. Dentro dessa categoria, alguns grupos de peixes se destacam, como o matrinxã, Brycon amazonicus (SPIX et al., 1829), que está entre as dez espécies mais desembarcadas e comercializadas na região do médio Rio Solimões (FERRAZ et al., 2016). Existe um padrão sazonal de produção e produtividade dessa espécie, com uma possível influência do pulso de inundação sobre estes parâmetros (FERRAZ et al., 2012).

Esta espécie de peixe possui desova total, fecundação externa e forma grandes cardumes que se deslocam em direção ao encontro dos rios de águas brancas para realização da desova nos períodos de elevação do nível fluviométrico no sistema fluvial Solimões/Amazonas. Ou seja, são espécies com comportamentos sazonais, que se enquadram nos padrões descritos por Goulding (1980).

As larvas de B. amazonicus foram observadas derivando pelo leito do rio em outras partes da Amazônia Central, principalmente próximos as suas margens, sendo posteriormente dispersas nas áreas de inundação adjacentes por meio dos canais e paranás presentes na planície Amazônica (MOUNIC-SILVA et al., 2013; ZACARDI, 2014), o que permite a estes organismos acesso a novos habitats, oferecendo ambientes variados de berçário e crescimento, além de áreas de proteção para a sobrevivência durante a fase inicial de desenvolvimento (JUNK et al., 1989; ZACARDI, 2014).

Porém, ações antropogênicas de escala local como a sobre-explotação dos recursos pesqueiros, uso inadequado do solo com remoção da cobertura vegetal e das matas ciliares, alterações em escala regional com a construção de hidrelétricas localizadas em diversos trechos da bacia Amazônica, sem contar as modificações continuas nos canais principais e áreas de várzeas dos rios, representam grandes ameaças para a ictiofauna amazônica.

Esses fatores devem ser levados em consideração, no que se refere ao desenvolvimento de políticas ambientais voltadas para a preservação e sustentabilidade dos recursos pesqueiros. Nesta perspectiva, a sustentabilidade da pesca comercial centralizada no médio Amazonas depende de um melhor conhecimento acerca dos seus estoques pesqueiros mais importantes (SANTOS et al., 2005), incluindo também o conhecimento sobre os padrões de recrutamento e desenvolvimento dos primeiros estágios de vida das espécies de maior importância econômica para a região.

Neste contexto, a Reserva de Desenvolvimento Sustentável Mamirauá (RDSM) surge no cenário atual como potencial ferramenta na conservação da biodiversidade e manejo pesqueiro, pois pode prevenir o colapso de estoques (QUEIROZ et al., 2005) e permitir a continuidade da produção larval, protegendo regiões de grande importância como áreas de desova e berçário para grande diversidade de espécies de peixes.

Logo, a identificação dos principais habitats utilizados pelos peixes durante o ciclo inicial de vida, e a conectividade com estes ambientes são essenciais para a aplicação de medidas de manejo, visando a 
Distribuição de larvas de Brycon amazonicus (PISCES: BRYCONIDAE) no entorno da Reserva de Desenvolvimento Sustentável Mamirauá: base

CAJADO, R. A.; OLIVEIRA, L. S.; OLIVEIRA, C. C.; PONTE, S. C. S.; BITTENCOURT, S. C. S.; QUEIROZ, H. L.; ZACARDI, D. M.

conservação dos ecossistemas e estoques pesqueiros (BARLETTA et al., 2010), permitindo a identificação de possíveis áreas consideradas Essential Fish Habitat (EFHs) para as espécies, como proposto por Bilkovic et al. (2002), Ziober et al. (2012) e Zacardi et al. (2017), as quais são de fundamental importância para a gestão da pesca e dos recursos pesqueiros.

Assim, o presente estudo teve por objetivo ampliar o conhecimento sobre a ecologia das larvas de Brycon amazonicus na região do médio rio Solimões e baixo rio Japurá, além de verificar a importância dos ambientes marginais para o recrutamento biológico dessa espécie, fornecendo informações importantes que podem ser utilizadas para auxiliar o manejo sustentável e a conservação desse recurso pesqueiro na Amazônia Central.

\section{MATERIAIS E MÉTODOS}

\section{Área de estudo}

O estudo foi realizado em 16 estações de amostragens, situadas no trecho médio do Rio Solimões e Baixo Rio Japurá, distribuídas em áreas próximas a bocas de canais de lagos ( $\mathrm{P} 1, \mathrm{P} 2$, P9 e P10), barrancos (P3, P4, P11 e P12), bancos de areia (P5, P6, P13 e P14) e canal central dos rios (P7, P8, P15, e P16), no entorno da Reserva de Desenvolvimento Sustentável Mamirauá $\left(03^{\circ} 08^{\prime} \mathrm{S}, 64^{\circ} 45^{\prime} \mathrm{W}\right.$ e $\left.2^{\circ} 36^{\prime} \mathrm{S}, 67^{\circ} 13^{\prime} \mathrm{W}\right)$, no estado do Amazonas, conforme a figura 1. As regiões marginais destes dois rios estão sujeitas a inundações periódicas, de intensidade que variam de acordo com os ciclos anuais das precipitações pluviométricas, na enchente, existe maior quantidade de chuvas e menor amplitude térmica.

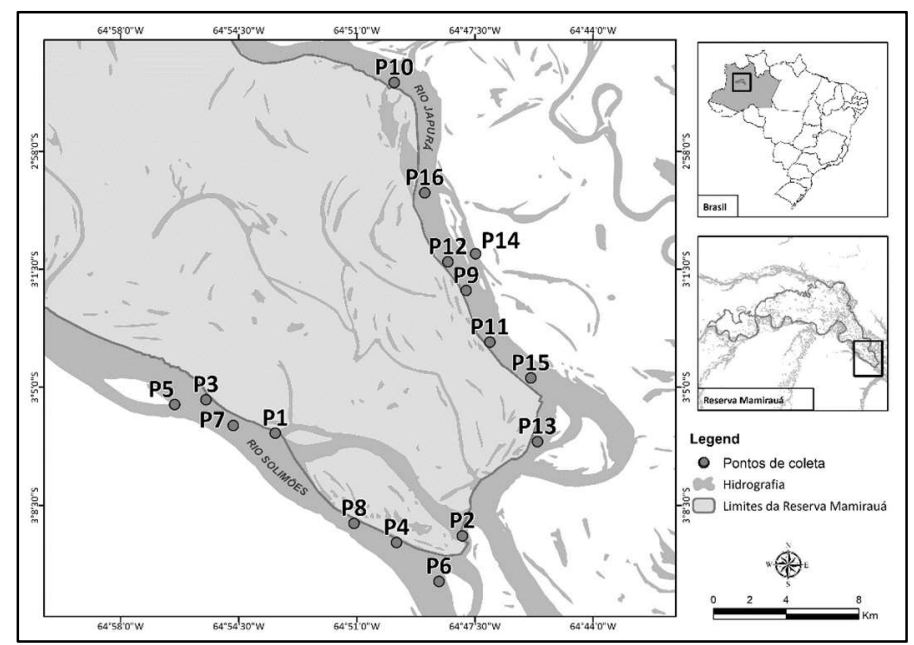

Figura 1: Localização da área de estudo, com destaque para estações de amostragem no entorno da Reserva de Desenvolvimento Sustentável Mamirauá (RDSM), Amazonas, Brasil. Fonte: Zacardi (2014).

\section{Coleta do Material Biológico}

As amostragens ocorreram em quatro momentos do ciclo hidrológico, representando os momentos de enchente (janeiro/fevereiro), cheia (maio/junho), vazante (julho/agosto) e seca (outubro/novembro), de 2011, por meio de arrastos horizontais em dois estratos da coluna d’água (subsuperfície e profundidade aproximadamente 8 metros), utilizando-se rede de plâncton cônico-cilíndrica (malha de $300 \mu \mathrm{m}$ ), com fluxômetro acoplado, para obter o volume de água volume de água filtrada. Para as coletas de profundidade, 
Distribuição de larvas de Brycon amazonicus (PISCES: BRYCONIDAE) no entorno da Reserva de Desenvolvimento Sustentável Mamirauá: base

CAJADO, R. A.; OLIVEIRA, L. S.; OLIVEIRA, C. C.; PONTE, S. C. S.; BITTENCOURT, S. C. S.; QUEIROZ, H. L.; ZACARDI, D. M.

a rede foi equipada com um sistema manual de abrir e fechar no fundo do rio, estabilizada por um peso de $12 \mathrm{~kg}$ e tracionada verticalmente.

Os arrastos tiveram duração de aproximadamente cinco minutos em todas as estações de amostragem e foram realizados com embarcação local em velocidade baixa e constante, no sentido oposto ao da correnteza, para garantir maior eficiência na captura, obedecendo a uma de distância de 5 a 15 metros das margens. Um total de 64 amostras foram coletadas para cada fase do ciclo hidrológico, totalizando 256 amostras ao final do estudo. Após cada arrasto, o material biológico foi fixado a bordo com solução formalina a $10 \%$, e acondicionado em potes de polietileno de $500 \mathrm{ml}$ devidamente etiquetados e levados para análise no Laboratório de Ecologia do Ictioplâncton, da Universidade Federal do Oeste do Pará, Santarém (PA).

\section{Processamento das amostras}

No laboratório, as amostras foram triadas, separando as larvas de peixes do material em suspensão, detrito e do plâncton total, com auxílio de pinças e placas de Petri, sob microscópio estereoscópico, e posteriormente as larvas foram quantificadas e identificadas ao menor nível taxonômico possível, tendo como base bibliografias especializadas.

Para a determinação do desenvolvimento larval foi considerado quatro estágios: larval vitelino, préflexão, flexão e pós-flexão, baseando-se no grau de flexão da seção terminal da notocorda, durante o desenvolvimento da nadadeira caudal, assim como proposto por Nakatani et al. (2001). Os espécimes identificados foram armazenados em frascos de vidro contendo formalina a $4 \%$ e etiquetados, para constituir uma coleção de referência no laboratório. O material biológico analisado foi coletado por meio da autorização de número 23741-1, expedida com base na Instrução Normativa no154/2007 e emitida pelo Sisbio/ICMBio/MMA.

\section{Análise dos dados}

Para as análises de variação na distribuição, a densidade de larvas foi padronizada para um volume de $100 \mathrm{~m}^{3}$ de água filtrada (Indivíduo/100m³), de acordo com Tanaka (1973) modificado por Nakatani et al. (2001). A densidade média (D) foi obtida através da equação: $D=d / N$, em que: $d=$ densidade de organismos coletados; $\mathrm{N}$ = número de amostragens realizadas.

Para verificar a existência de diferenças significativas na densidade média entre as unidades de habitat (canais de lagos, barrancos, bancos de areia e canal central dos rios), períodos (diurno e noturno) e estratos da coluna d'água (subsuperfície e fundo), aplicou-se ANOVA bifatorial considerando os habitats, os períodos e os estratos de amostragens como fatores. Para atingir os pressupostos de normalidade dos dados e homogeneidade das variâncias as densidades médias foram log-transformadas $(\log 10(x+1))($ PETERS, 1986). O Teste a posteriori de Tukey foi aplicado sempre que diferenças significativas $(P<0,05)$ foram detectadas, utilizando o software STATISTICA 7.0. 


\section{DISCUSSÃO TEÓRICA}

No total, foram coletadas 77.250 larvas de peixes capturadas, nos trechos do médio Rio Solimões e Baixo Rio Japurá. A ordem Characiformes contribuiu com $89 \%$ das larvas capturadas. Dentre as larvas pertencentes ao grupo dos Characiformes, no Médio Rio Solimões, e desse total, 148 foram identificadas como larvas de B. amazonicus (matrinxã). Com relação aos dados referentes ao trecho do baixo rio Japurá, 162 larvas foram identificadas como B. amazonicus, totalizando 310 indivíduos. Ressalta-se que em ambos os rios as larvas de matrinxã tiveram ocorrência registrada apenas durante o momento de enchente (janeiro/fevereiro), na área de estudo.

A densidade média de larvas não apresentou diferença significativa (ANOVA, $p>0,05$ ) entre os dois rios e os habitats analisados, no entanto foram constatados que as larvas de $B$. amazonicus apresentaram distribuição distinta entre os dois rios estudados. Para o trecho médio do rio Solimões os maiores valores de densidade registrados foram em áreas próximas a barrancos $\left(1,95 \mathrm{ind} . / 10 \mathrm{~m}^{3}\right)$. Já no trecho baixo do rio Japurá, registrou-se a maior densidade em áreas de confluência dos canais de lagos com o rio $\left(0,73\right.$ ind. $\left./ 10 \mathrm{~m}^{3}\right)$, como se pode ver na figura 2 .

As larvas de $B$. amazonicus tiveram ocorrência registrada em fases avançadas de desenvolvimento (flexão - 98,39\% e pós-flexão - 1,61\%) ao longo dos diversos habitats amostrados. Sendo que foram identificados $76 \%$ dos indivíduos com alimento no estômago, e a maioria (62\%) exibia como conteúdo larvas de Characiformes.

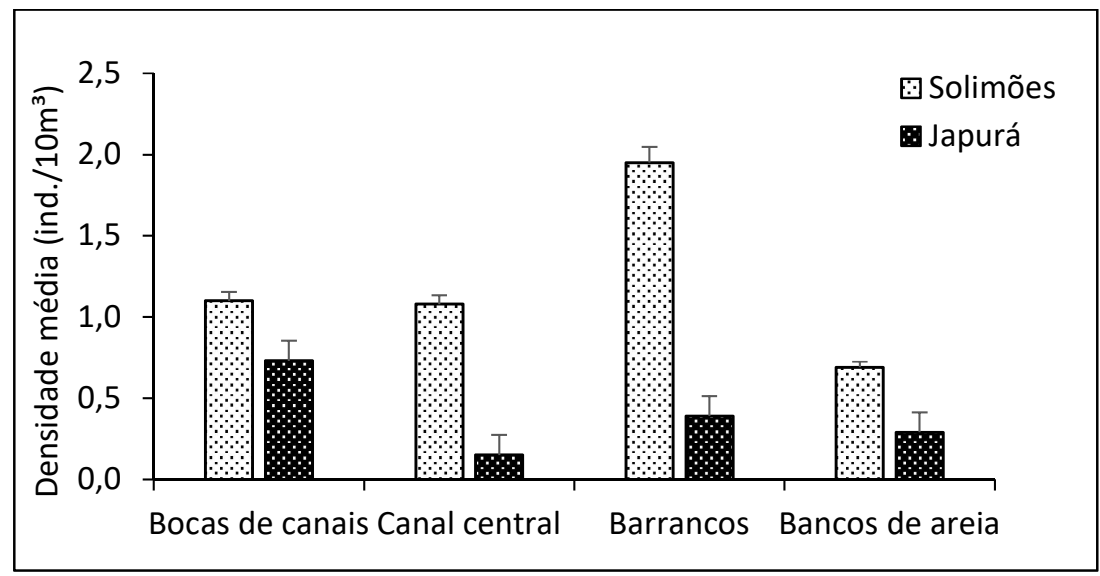

Figura 2: Densidade de larvas de B. amazonicus (ind./10 ${ }^{3}$ ) capturadas nos habitats presentes ao longo dos trechos do médio rio Solimões e baixo rio Japurá, no entorno da RDSM, Amazônia Central (barra= erro padrão).

Com relação aos períodos diurno e noturno, foram observadas diferenças significativas entre as amostras (ANOVA; $\mathrm{F}=2,99 ; \mathrm{p}=<0,01$ ), com os maiores valores registrados no período noturno para o rio Solimões $\left(3,74\right.$ ind. $/ 10 \mathrm{~m}^{3}$ ) e baixo Japurá $\left(2,33\right.$ ind. $/ 10 \mathrm{~m}^{3}$ ) (Teste de Tukey, $p<0,05$ ) (figura 3), evidenciando uma possível migração vertical no comportamento das larvas de B. amazonicus, confirmando uma periodicidade diária na deriva das larvas desta espécie. 


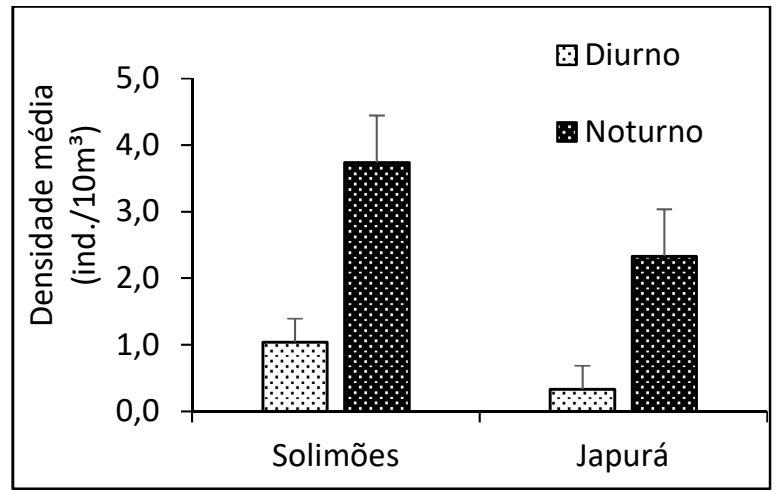

Figura 3: Densidade de larvas de B. amazonicus (ind./10m ${ }^{3}$ ) capturadas durante os períodos diurno e noturno, nos trechos do médio rio Solimões e baixo rio Japurá, no entorno da RDSM, Amazônia Central (barra= erro padrão).

Para a análise da variação entre os diferentes estratos da coluna d'água, os resultados mostraram diferença significativa (ANOVA; $F=5,24 ; p=0,02$ ), entre as densidades de larvas capturadas em profundidade e subsuperfície, com as maiores abundâncias registradas na subsuperfície, propostas na figura 4, confirmadas pelo teste de Tukey $(p<0,05)$, indicando este estrato como o micro-habitat onde há maiores variações na velocidade do rio e alta turbulência mais utilizado pelas larvas.

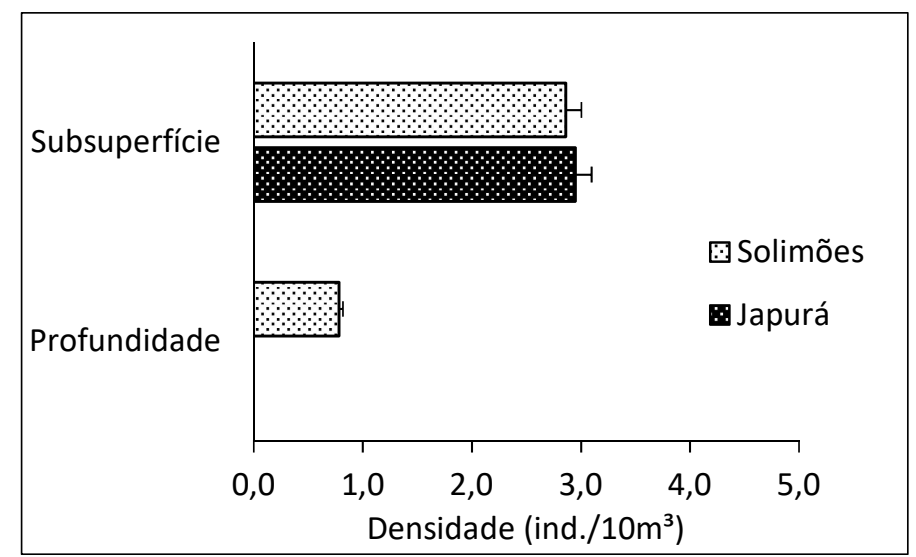

Figura 4: Densidade de larvas de B. amazonicus (ind./10 ${ }^{3}$ ) capturadas nos diferentes estratos da coluna d'água, nos trechos do médio rio Solimões e baixo rio Japurá, no entorno da RDSM, Amazônia Central (barra= erro padrão).

Numa série histórica, observa-se que a presença de larvas no canal fluvial do sistema Solimões/Amazonas oscila quantitativamente e qualitativamente dentro de um ciclo hidrológico completo, de acordo com Araújo-Lima et al. (1998), e que no início da subida das águas dos Rios Solimões e Japurá, que se estende de dezembro até abril, há maior disponibilidade de larvas de peixes do que nos meses de cheia, seca e vazante (ZACARDI, 2014). Isto porque o desenvolvimento gonadal e a reprodução da maioria dos peixes Amazônicos estão relacionados com as flutuações no nível fluviométrico, principalmente aos períodos de inundação, pois o transbordamento da água na planície proporciona abrigo e uma elevada produção de alimento adequado às fases iniciais de desenvolvimento dos peixes (SÁNCHEZ-BOTERO et al., 2001), favorecendo o desenvolvimento das larvas e juvenis.

Apesar de não ter sido observado diferença significativa quanto à variação espacial de larvas de $B$. amazonicus, constatou-se que elas não são distribuídas uniformemente em todas as feições do rio. As margens e as confluências do canal do rio com os canais de lagos são mais utilizadas pelas larvas de matrinxã, 
Distribuição de larvas de Brycon amazonicus (PISCES: BRYCONIDAE) no entorno da Reserva de Desenvolvimento Sustentável Mamirauá: base ecológica para manejo

CAJADO, R. A.; OLIVEIRA, L. S.; OLIVEIRA, C. C.; PONTE, S. C. S.; BITTENCOURT, S. C. S.; QUEIROZ, H. L.; ZACARDI, D. M.

independentemente do trecho de rio estudado, seja no médio Solimões ou baixo Japurá, revelando uma estratégia reprodutiva da espécie, sendo muito importantes nos mecanismos de dispersão e desenvolvimento inicial das larvas de matrinxã, devido à maior hidrodinâmica local que auxilia o carreamento das larvas para as margens inundadas (regiões mais lênticas) em que ocorrem maior sedimentação e acúmulo de material forrageiro. As larvas de matrinxã também ocupam as áreas de vegetação, onde encontram alimento e abrigo (LEITE, 2000; MOUNIC-SILVA et al., 2013).

Os matrinxãs se reproduzem no final da vazante (LIMA et al., 2004; GRANADO-LORENCIO et al., 2005). Essa estratégia adotada é para alcançar os tamanhos apropriados para capturar suas presas que predominarão durante o aumento do nível das águas do rio. Neste momento de enchente, as larvas de $B$. amazonicus se aproveitam do seu maior tamanho, para se alimentarem de larvas de outras espécies de Characiformes (LEITE et al., 2006), conseguindo completar seu ciclo inicial nas áreas marginais e no canal do rio principal.

Neste sentido, as estratégias de desova dos peixes estão diretamente associadas aos processos hidrodinâmicos, aspectos tróficos e com o pulso de inundação, sendo os principais fatores responsáveis pela dispersão diferenciada das larvas no corpo hídrico (PONTES et al., 2016). Desta maneira os padrões de distribuição das larvas nestes habitats podem ser definidos a partir do efeito da ação conjunta de desovas próximas as margens e bocas de canais e da ação das correntes sobre a dispersão larval próxima às zonas de erosão (barrancos) (OLIVEIRA et al., 1998).

A maior captura de exemplares durante o período noturno confirma a periodicidade diária de larvas, comportamento observado e registrado também por Baumgartner (2001). Esse padrão diário provavelmente ocorre devido à evitabilidade da rede de plâncton pelos organismos, em períodos de maior luminosidade, principalmente em estágios de desenvolvimento larval mais avançado (SHENKER et al., 1993), ou pela utilização da superfície da coluna d'água, durante a noite, como área de refúgio contra predadores e alimentação (PICAPEDRA et al., 2015), e durante o dia ficam mais próximas do fundo ou dispersas na coluna d'água como observado por Araújo-Lima et al. (2001) e Pontes et al. (2016), maximizando a sobrevivência da prole, explicando a ocorrência da elevada densidade de larvas na superfície, durante as coletas no período noturno.

De acordo com Zacardi et al. (2016), muitas espécies de peixes adquiriram durante sua evolução mecanismos de migração vertical na coluna d'água, que lhe conferem maior segurança e probabilidade de sobrevivência. Para Zacardi, (2015) estes mecanismos consistem na submersão das larvas durante o dia (período mais susceptível à predação) e emersão na coluna d'água durante a noite (período em que o risco de predação e o estresse térmico próximo à superfície são menores e a oferta de alimentos é maior).

A maior densidade de larvas encontrada na subsuperfície da água à noite é uma característica que tende a aumentar à medida que as larvas se desenvolvem. Esse comportamento pode ser um mecanismo para minimizar a competição intraespecífica (SANTIN et al., 2004; OSTERGAARD et al., 2005), indicando um comportamento migratório ativo das larvas (OLIVEIRA et al., 1998). Fato que pode estar associado às baixas 
Distribuição de larvas de Brycon amazonicus (PISCES: BRYCONIDAE) no entorno da Reserva de Desenvolvimento Sustentável Mamirauá: base ecológica para manejo

CAJADO, R. A.; OLIVEIRA, L. S.; OLIVEIRA, C. C.; PONTE, S. C. S.; BITTENCOURT, S. C. S.; QUEIROZ, H. L.; ZACARDI, D. M.

quantidades de larvas de matrinxã capturadas, uma vez que larvas mais desenvolvidas conseguem fugir (escapar) mais facilmente do aparelho de captura.

Dentre as larvas de $B$. amazonicus, apenas 5 exemplares foram capturados em estágio ontogênico de pós-flexão, as demais encontravam-se em estágio de flexão. A presença das larvas nesses estágios de desenvolvimento indica que as larvas de matrinxã utilizam as regiões marginais como área de criação e alimentação, conseguindo completar todo o seu ciclo inicial de vida no canal e nas margens ao longo do rio, sendo independentes das áreas de várzea, diferindo das demais espécies de Characiformes migradores. Estes fatores são indicativos da existência de um grande número de EFHs ao longo do trecho médio do Rio Solimões e Baixo Japurá, incluindo os seus ambientes de várzea (canais e lagos), que são importantes para a manutenção dos estoques pesqueiros da Amazônia.

Bialetzki et al. (1999), Baumgartner et al. (1997) e Baumgartner (2001) também encontraram maiores abundâncias de larvas de peixes derivando na subsuperfície da coluna d'água em dois canais do Rio Paraná e afluentes, comportamento semelhante ao encontrado no presente trabalho, indicando um comportamento ativo das larvas de B. amazonicus neste micro-habitat, onde há maiores variações na velocidade do rio, alta turbulência e maiores possibilidades de conseguir alimento, uma vez que predam larvas de Characiformes recém-eclodidas.

Neste sentido, os resultados indicam que o trecho estudado é uma área importante para o desenvolvimento inicial de B. amazonicus, servindo como área de dispersão e alimentação das larvas, e que os diversos habitats presentes dos ecossistemas fluviais no entorno da RDSM são relevantes para a manutenção e recuperação desses estoques pesqueiros, sendo necessária a permanência de sua integridade ambiental visando à conservação e a sustentabilidade das populações de espécies de interesse econômico para a pesca. Portanto, estas áreas no trecho médio do Rio Solimões e Baixo Rio Japurá, consistem em um ambiente com status de berçário para espécies comercialmente explotadas na Amazônia Central.

\section{CONCLUSÕES}

Analisando a distribuição das larvas de B. amazonicus nos trechos do médio Rio Solimões e Baixo Japurá fica evidente que a estrutura das larvas desses peixes é influenciada por vários fatores intrínsecos e extrínsecos. Assim, há de se considerar a importância dos diferentes, habitats e micro-habitats analisados, destacando que as regiões de várzea no entorno da Reserva de Desenvolvimento Sustentável Mamirauá é uma área importante como criadouro natural, servindo como local de crescimento e alimentação nesta fase inicial do desenvolvimento da matrinxã, tornando-se fundamental a sua conservação, buscando garantir a manutenção dos estoques pesqueiros da região.

\section{REFERÊNCIAS}

ARAÚJO-LIMA, C. A. R. M.; OLIVEIRA, E. C.. Transport of larval fish in the Amazon. Journal of fish Biology, v.53, p.297-306, 1998. DOI: https://doi.org/10.1111/j.10958649.1998.tb01033.x
ARAÚJO-LIMA, C. A. R. M.; SILVA, J. V. V.; PETRY, P.; OLIVEIRA, E. C.; MOURA, S. M. L.. Diel variation of larval fish abundance in the Amazon and Rio Negro. Brazil. Journal of Biology, v.61, n.3, p.357-362, 2001. DOI:

http://doi.org/10.1590/S1519-69842001000300003 
BARLETTA, M.; JAUREGUIZAR, A. J.; BAIGUN, C.; FONTOURA, N. F.; AGOSTINHO, A. A.; ALMEIDA-VAL, V. M. F.; VAL, A. L.; TORRES, R. A.; JIMENES-SEGURA, L. F.; GIARRIZZO, T.; FABRÉ, N. N.; BATISTA, V. S.; LASSO, C.; TAPHORN, D. C.; COSTA, M. F.; CHAVES, P. T.; VIEIRA, J. P.; CORRÊA, M. F. M.. Fish and aquatic habitat conservation in South America: a continental overview with emphasis on neotropical systems. Journal of Fish Biology, v.76, p.2118-2176, 2010. DOI:

https://doi.org/10.1111/j.10958649.2010.026x

BAUMGARTNER, G.. Determinação dos locais de desova e criadouros naturais de peixes e influência dos fatores abióticos sobre a abundância de larvas no alto Rio Paraná, Brasil. Tese (Doutorado) - Universidade Estadual de Maringá, Maringá, 2001.

BAUMGARTNER, G.; NAKATANI, K.; CAVICCHIOLI, M.; BAUMGARTNER, M. D. S. T.. Some aspects of the ecology of fishes larvae in the floodplain of the high Paraná River, Brazil. Revista Brasileira de Zoologia, Curitiba, v.14, n.3, p.551-563, 1997. DOI:

http://doi.org/10.1590/S010181751997000300005

BIALETZKI, A.; SANCHES, P. V.; CAVICCHIOLI, M.; BAUMGARTNER, G.; RIBEIRO, R. P.; NAKATANI, K.. Drift of ichthyoplankton in two channels of the Paraná River, between Paraná and Mato Grosso do Sul States, Brazil. Brazilian Archives of Biology and Technology, Curitiba, v.42, n.1, p.53-60, 1999.

BILKOVIC, D. M.; HERSHNER, C. H.; OLNEY, J. E.. Macroscale assessment of American shad spawning and nursery habitat in the Mattaponi and Pamunkey Rivers, Virginia. North American Journal of Fisheries Management, v.22, n.4, p.1176-1192, 2002. DOI: https://doi.org/10.1577/15488675(2002)022<1176:MAOASS $>2.0 . \mathrm{CO} ; 2$

FERRAZ, P.; BARTHEM, R.. Estatística do Monitoramento do Desembarque Pesqueiro na Região de Tefé: Médio Solimões. Tefé: IDSM, 2016.

FERRAZ, P.; LIMA, D.; AMARAL, E.. Estatística do monitoramento do desembarque pesqueiro na região de Tefé: Médio Solimões: Os primeiros 16 anos (1992-2007). Tefé: IDSM, 2012.

GOULDING, M.. The fishes and the forest: explorations in Amazonian natural history. Berkeley: UCP, 1980.

GRANADO-LORENCIO, C.; LIMA, C. R. M.; LOBÓN-CERVIÁ, J.. Abundance-distribution relationships in fish assembly of the Amazonas floodplain lakes. Ecography, v.28, n.4, p.515-520, 2005. DOI: https://doi.org/10.1111/i.09067590.2005.04176.x

JUNK, W. J.; BAYLEY, P. B.; SPARKS, R. E.. The flood pulse concept in river floodplain systems. Canadian Special Publication of Fisheries and Aquatic Science, v.106, p.110127, 1989.

LEITE, R. G.. Alimentação e fontes autotróficas de energia utilizadas pelas larvas de peixes do rio Solimões/Amazonas e suas áreas inundáveis. Tese (Doutorado) Instituto Nacional de Pesquisas da Amazônia, Manaus, 2000.
LEITE, R. G.; SILVA, J. V. V.; FREITAS, C. E.. Abundância e distribuição das larvas de peixes no Lago Catalão e no encontro dos rios Solimões e Negro, Amazonas, Brasil. Acta Amazônica, v.36, n.4 p.557-562, 2006.

LIMA, A. C; ARAÚJO-LIMA, C. A. R. M.. The distribution of larval and juvenile fishes in Amazonian rivers of different nutrient status. Freshwater Biology, v.49, p.1-14, 2004. DOI: https://doi.org/10.1111/i.1365-2427.2004.01228.x

MOUNIC-SILVA, C. E.; LEITE, R. G.. Abundance of young-ofthe-year migratory Characiforms in floodplain areas of the middle Solimões-Amazon River at flooding 2007/2008. Journal of Applied Ichthyology, v.29, n.1, p.118124, 2013. DOI: https://doi.org/10.1111/j.1439$\underline{0426.2012 .02047 . x}$

NAKATANI, K.; AGOSTINHO, A. A.; BAUMGARTNER, G.; BIALETZKI, A.; SANCHES, P. V.; MAKRAKIS, M. C. \& PAVANELLI, C. S.. Ovos e larvas de peixes de água doce: desenvolvimento e manual de identificação. Maringá: EDUEM, 2001.

OSTERGGAARD P.; MUNK P.; JANEKARN, V.. Contrasting feeding patterns among species of fish larvae from the tropical Andaman Sea. Marine Biology, v.146, p.595-606, 2005. DOI: http://doi.org/10.1007/s00227-004-1458-8

PETERS, R. H.. The role of prediction in limnology. Limnology and Oceanography, v.31, n.5, p.1143-1159, 1986. DOI: https://doi.org/10.4319/lo.1986.31.5.1143

PICAPEDRA, P. H. S.; LANSAC-TÔHA, F. A.; BIALETZKI, A.. Diel vertical migration and spatial overlap between fish larvae and zooplankton in two tropical lakes, Brazil. Brazilian Journal of Biology, v.75, n.2, p.352-361, 2015. DOI: http://doi.org/10.1590/1519-6984.13213

PONTES, S. C. S.; FERREIRA, L. C.; BITTENCOURT, S. C. S.; QUEIROZ, H. L.; ZACARDI, D. M.. Variação espacial e temporal das larvas de Triportheus (Characiformes, Triportheidae), no médio Rio Solimões, Amazônia Central, Brasil. Acta of Fisheries and Aquatic Resources, v.4, p.71-81, 2016. DOI: http://doi.org/10.2312/Actafish.4.2.71-81

QUEIROZ, H. L. A.. Reserva de Desenvolvimento Sustentável Mamirauá. Revista de Estudos Avançados, v.19, n.54, 2005 QUEIROZ, H. L.; CRAMPTON, W. G. R.. Estratégias de Manejo de Recursos Pesqueiros em Mamirauá. Brasília: MCT, 1999.

SÁNCHEZ-BOTERO J. I.; ARAÚJO-LIMA, A. C. R. M.. As macrófitas aquáticas como berçário para a ictiofauna da várzea do rio Amazonas. Acta Amazônica, v.31, n.3, p.437447, 2001. DOI: http://doi.org/10.1590/180943922001313447

SANTIN, M.; BIALETZKI, A.; NAKATANI, K.. Mudanças ontogênicas no trato digestório e dieta de Apareiodon affinis (Steindachner, 1879) (Osteichthyes, Parodontidae). Acta Scientiarum Biological Sciences, v.26, n.3, p.291-298, 2004. DOI: http://doi.org/10.4025/actascibiolsci.v26i3.1542

SANTOS, G. M.; SANTOS, A. C. M.. Sustentabilidade da pesca na Amazônia. Estudos avançados, v.19, n.54, p.165182, 2005. DOI: http://doi.org/10.1590/S010340142005000200010 
Distribuição de larvas de Brycon amazonicus (PISCES: BRYCONIDAE) no entorno da Reserva de Desenvolvimento Sustentável Mamirauá: base ecológica para manejo

CAJADO, R. A.; OLIVEIRA, L. S.; OLIVEIRA, C. C.; PONTE, S. C. S.; BITTENCOURT, S. C. S.; QUEIROZ, H. L.; ZACARDI, D. M.

SHENKER, J. M.; MADDOX, E. D.; WISHINSKI, E.; PEARL, A.; THORROLD, S. R.; SMITH, N.. Onshore transport of settlement-stage Nassau grouper Epinephelus striatus and other fishes in Exuma Sound, Bahamas. Marine Ecology Progress Series, v.98, n.1, p.31-43, 1993. DOI: http://doi.org/10.3354/meps098031

TANAKA, S.. Stock assessment by means of ichthyoplankton surveys. FAO: Fisheries Technical Paper, v. 122, p.33-51, 1973.

WELCOMME, R.. World prospects for floodplain fisheries. Ecohydrology \& Hydrobiology, v.8, n.4, p.169-182, 2008. DOI: https://doi.org/10.2478/v10104-009-0013-0

ZACARDI, D. M., BITTENCOURT, S. C. S.; NAKAYAMA, L. QUEIROZ, H. L.. Distribution of economically important fish larvae (Characiformes, Prochilodontidae) in the Central Amazonia, Brazil. Fisheries Management and Ecology, p.19, 2017. DOI: http://doi.org/10.1111/fme.12222

ZACARDI, D. M., PONTE, S. C. S.. Padrões de distribuição e ocorrência do ictioplâncton no médio Rio Xingu, bacia
Amazônica, Brasil. Revista em Agronegócio e Meio

Ambiente, v.9, p.949-972, 2016. DOI:

http://doi.org/10.17765/2176-9168.2016v9n4p949-972

ZACARDI, D. M.. Abundância e distribuição espaço-temporal de ovos e larvas de peixes de interesse econômico no Médio Rio Solimões e Baixo Rio Japurá, Amazônia Central, Brasil. Tese (Doutorado em Ciência Animal) - Universidade Federal do Pará, Belém, 2014.

ZACARDI, D. M.. Variation and abundance of ichthyoplankton in tidal channels on Extreme North Brazil. Biota Amazônica, v.5, n.1, p.43-52, 2015. DOI: http://doi.org/10.18561/21795746/biotaamazonia.v5n1p43-52

ZIOBER, S. R.; BIALETZKI, A.; MATEUS, L. A. F.. Effect of abiotic variables on fish eggs and larvae distribution in headwaters of Cuiabá River, Mato Grosso State, Brazil. Neotropical ichthyology, v.10, n.1, p.123-132, 2012. DOI: http://doi.org/10.1590/S167962252012000100012

A CBPC - Companhia Brasileira de Produção Científica (CNPJ: 11.221.422/0001-03) detém os direitos materiais desta publicação. Os direitos referem-se à publicação do trabalho em qualquer parte do mundo, incluindo os direitos às renovações, expansões e disseminações da contribuição, bem como outros direitos subsidiários. Todos os trabalhos publicados eletronicamente poderão posteriormente ser publicados em coletâneas impressas sob coordenação da Sustenere Publishing, da Companhia Brasileira de Produção Científica e seus parceiros autorizados. Os (as) autores (as) preservam os direitos autorais, mas não têm permissão para a publicação da contribuição em outro meio, impresso ou digital, em português ou em tradução. 